\title{
Scientific Control
}

National Cancer Institute

\section{Source}

National Cancer Institute. Scientific Control. NCI Thesaurus. Code C64355.

Methodology that tests integrity in experiments by isolating variables as dictated by the scientific method in order to make a conclusion about such variables. In a controlled experiment, two virtually identical experiments are conducted, but the factor being tested is varied in only one of them. This serves to isolate any causal phenomena. 